\title{
On Totally Real Cubic Fields
}

\author{
By Veikko Ennola and Reino Turunen
}

\begin{abstract}
The authors have constructed a table of the 26440 nonconjugate totally real cubic number fields of discriminant $D<500000$ thereby extending the existing table of fields with $D<100000$ by I. O. Angell [1]. Serious defects in Angell's table are pointed out. For each field, running number, discriminant, coefficients of a generating polynomial, integral basis, class number, and a fundamental pair of units are listed. The article contains statistics about the following subjects: distribution of class numbers; fields in which every norm-positive unit is totally positive; nonconjugate fields with the same discriminant; fields with noncyclic class group. The fields are tabulated by means of a method due to Davenport and Heilbronn [7], [8] which leads to a unique normalized generating polynomial. The given units are chosen so that the fundamental parallelogram of the unit lattice determined by the corresponding vectors in the logarithmic space is reduced.
\end{abstract}

1. Introduction. A table of totally real cubic fields of discriminant $D<100000$ has previously been constructed by I. O. Angell [1]. In this article we shall describe the construction of an extended table for $D<500000$. It has been deposited in the Mathematics of Computation's UMT-depository.

The motive for this work stems firstly as a by-product from the first author's wish to investigate certain parametric families of totally real cubic fields with small fundamental pairs of units and large class number, and secondly from the fact that serious defects have been discovered in Angell's table. A list of such defects which have come to our attention is as follows.

(1) There are 11 fields missing. These fields have discriminants 25717, 32404 , $35996,37108,37133,38905,39992,43165,43173,43176,95484$. The omission of the first field has been discovered by Franz Halter-Koch and has evidently been corrected in later versions of the table. The omission of the other ten fields has previously been independently discovered by Llorente and Oneto [15].

(2) The field with discriminant 88588 appears twice.

(3) In "Appendix of units with large coefficients" there are two errors. For $D=81377$, the first number, and for $D=82657$, the second one are not units: they both have norms divisible by 10 .

(4) In the statistics referring to the class numbers [1, p. 186] there are several mistakes as discovered by Llorente and Oneto [15]. They give a revised version of these statistics which, however, is not fully in accordance with our results (see Section 7 below for details).

Received April 11, 1983; revised March 14, 1984.

1980 Mathematics Subject Classification. Primary 12A30, 12-04, 12A45, 12A50.

1985 American Mathematical Society (0)25-5718/85\$1.00+\$.25 per page 
(5) Angell's adaptation of the Voronoi algorithm does not necessarily produce a fundamental pair of units (e.g., in the case $D=39601=199^{2}$, the given units together with -1 generate a subgroup of index 3 in the group of units). This observation was made by M. N. Gras.

The method we have used in order to tabulate the fields is due to Davenport and Heilbronn [7], [8]. It is different from that of Angell, but a somewhat similar approach has previously been used by Llorente and Oneto [15]. As was shown by Davenport and Heilbronn, there exists a bijective discriminant-preserving map of the set of triplets of conjugate totally real cubic fields onto a subset $\mathscr{R}$ of the set of reduced integral primitive irreducible binary cubic forms. Here we have to modify slightly the classical concept of a reduced cubic form [10, Chapter XII] in order to have just a single one contained in each equivalence class. The local conditions of Davenport and Heilbronn, which are necessary and sufficient for a reduced form to belong to $\mathscr{R}$, are given below in a simplified form.

In the search for all forms in $\mathscr{R}$ with discriminants in a given range it is useful to have stringent limitations for their coefficients. We therefore present a collection of best possible inequalities satisfied by those coefficients.

From a form in $\mathscr{R}$ we construct a monic cubic polynomial with integral coefficients in an obvious manner. A polynomial obtained in that way is called a normalized cubic polynomial (NCP) and its zeros are termed normalized primitive elements (NPE). Hence, a noncyclic field is generated by a unique naturally defined NPE, while a cyclic field contains three such elements. We show that if $\alpha$ is an NPE of a field $K$, the conjugates of $\alpha$ have least standard deviation among all irrational algebraic integers $\beta$ of $K$, and that this property is shared only by certain particular $\beta$ 's naturally related with $\alpha$. If $K$ is cyclic then, apart from sign, the NPE's are equal to the Gaussian periods for a generating cubic character of $K$.

Our method thus leads automatically to a complete set of different fields: in particular, no Tschirnhausen transformation or any other means are needed to test the fields for being distinct. Moreover, an integral basis of $K$ and the value of the discriminant are readily at hand.

In order to compute the class number (class group structure) and a fundamental pair of units, we have used the classical Voronoi algorithm [9, Chapter IV]. It works very efficiently, the only drawback being the rather high degree of precision needed in a few cases due to the largeness of the units produced by the algorithm. In the unit lattice we have performed a reduction process to the effect that the final units to be listed in the table (called reduced units) are so chosen that the fundamental parallelogram of the lattice determined by the corresponding vectors in the logarithmic space is reduced. In this way we find a naturally defined fundamental pair of units, the choice of which is optimal in a certain sense.

For the extended range we give similar statistics as the one in [1] and [15] already discussed. The total number of nonconjugate fields with discriminants less than 500000 is 26440 giving the empirical density 0.05288 , whereas Davenport and Heilbronn [8] proved that the asymptotic value is $(12 \zeta(3))^{-1}=0.06933$. So the convergence is very slow as noted in [17].

At the end of the article, there are tables of fields in which every unit is totally positive or totally negative, of nonconjugate fields with the same discriminant, and 
of fields with noncyclic class group. The last property is a very rare one; we have encountered only 35 such cases.

All of the computations were done on the DEC-20 computer at the University of Turku, Finland.

We would like to thank Mme. M. N. Gras, Dr. Jukka Pihko, and Professors Harvey Cohn, H. J. Godwin, Franz Halter-Koch, and Jacques Martinet for their assistance and helpful comments. We are especially indebted to the members of the staff of the Computer Centre at the University of Turku for their cooperation. The work has been supported financially by the Academy of Finland.

For any cubic field $K$ we denote by $\mathcal{O}=\mathcal{O}_{K}$ the ring of integers of $K$. If $\beta \in K$, its conjugates are denoted either by $\beta, \beta^{\prime}, \beta^{\prime \prime}$ or by $\beta^{(i)}\left(i=0,1,2 ; \beta^{(0)}=\beta\right)$. The trace and norm of $\beta$ are $\operatorname{Tr}(\beta)=\beta+\beta^{\prime}+\beta^{\prime \prime}$ and $N(\beta)=\beta \beta^{\prime} \beta^{\prime \prime}$. We also write $N(\mathfrak{A})$ for the norm of a nonzero fractional ideal $\mathfrak{A}$ of $K$. The symbol $\square$ indicates the end of a proof.

2. Reduction of Binary Cubic Forms. We shall assume in the sequel that the binary cubic forms

$$
F(x, y)=a x^{3}+b x^{2} y+c x y^{2}+d y^{3}
$$

which we are dealing with are (i) integral, i.e., $a, b, c, d \in \mathbf{Z}$; (ii) primitive, i.e., $\operatorname{gcd}(a, b, c, d)=1$; (iii) irreducible in the ring $\mathbf{Q}[x, y]$; and (iv) have positive discriminant

$$
D(F)=b^{2} c^{2}-4 a c^{3}-4 b^{3} d-27 a^{2} d^{2}+18 a b c d .
$$

Accordingly, when speaking simply of cubic forms we always assume that the form is binary and that these conditions are satisfied. Equivalence of forms (both cubic and quadratic) and equivalence class are understood in the wide sense, i.e., homogeneous linear substitutions with integral coefficients and determinant $= \pm 1$ are admitted. If determinant $=+1$ is required, we expressly speak of proper equivalence and proper class. The cubic form (2.1) has the quadratic covariant

$$
H(x, y)=T x^{2}+U x y+V y^{2},
$$

where

$$
T=b^{2}-3 a c, \quad U=b c-9 a d, \quad V=c^{2}-3 b d .
$$

It is well-known that the quadratic form (2.3) is positive definite and its discriminant is

$$
U^{2}-4 T V=-3 D(F)<0 .
$$

According to the classical reduction theory of Arndt [2] and Hermite [13] the form (2.1) is called reduced iff (2.3) is a reduced quadratic form, i.e., either $-T<U \leqslant T$ $<V$ or $0 \leqslant U \leqslant T=V$. In the following theorem we introduce a slightly modified concept in order to obtain a unique representative for each class.

THEOREM 1. Every equivalence class $\mathscr{C}$ of binary cubic forms contains exactly one REDUCED FORM $F(x, y)=F_{\mathscr{C}}(x, y)$ satisfying one of the following conditions:

(i) $-T<U<T<V, a>0$, and either $b>0$ or $b=0$ and $d>0$,

(ii) $0<U=T<V, a>0$, and $b>3 a / 2$,

(iii) $0<U<T=V, a>0$, and $|d|>a$,

(iv) $0=U<T=V, a>0$, and $d<-a$,

(v) $0<U=T=V, a>0$, and $b>3 a / 2$. 
Proof. Let $\mathscr{C}$ be given. By the classical theory referred to above, we can pick a form $F(x, y) \in \mathscr{C}$ such that $-T<U \leqslant T<V$ or $0 \leqslant U \leqslant T=V$. Applying the substitution $x=-x^{\prime}, y=-y^{\prime}$ if necessary, we may suppose that $a>0$.

Case 1. $-T<U<T<V$. Clearly, either $F(x, y)$ or $F(x,-y)$ satisfies the conditions (i) and is thus the required $F_{\mathscr{C}}(x, y)$. Let $H_{\mathscr{C}}(x, y)$ denote the quadratic covariant of $F_{\mathscr{C}}(x, y)$. Suppose that $F_{1}(x, y)$ is another form in $\mathscr{C}$ satisfying (i) and let $H_{1}(x, y)$ denote its quadratic covariant. Since $F_{1}$ and $F_{\mathscr{C}}$ are equivalent, so are their quadratic covariants, and hence $H_{1}(x, y)$ is properly equivalent to $H_{\mathscr{C}}(x, y)$ or to $H_{\mathscr{C}}(x,-y)$. Since all three quadratic forms are reduced, either $H_{1}(x, y)=H_{\mathscr{C}}(x, y)$ or $H_{1}(x, y)=H_{\mathscr{C}}(x,-y)$. Let $\tau$ denote a substitution with determinant $=+1$ transforming $F_{1}(x, y)$ correspondingly into $F_{\mathscr{C}}(x, y)$ or $F_{\mathscr{C}}(x,-y)$ so that $\tau$ is an automorph of $H_{1}(x, y)$. By [11, p. 72, Theorem 57] $\tau$ is either the identity or $\left[x=-x^{\prime}, y=-y^{\prime}\right]$. The latter alternative is impossible because the leading coefficients are positive, and therefore $F_{1}(x, y)=F_{\mathscr{C}}(x, y)$ or $F_{\mathscr{C}}(x,-y)$. However, $F_{\mathscr{C}}(x,-y)$ does not satisfy (i). This proves the uniqueness of $F_{\mathscr{C}}$.

Case 2. $U=T<V$. The quadratic covariant of the form $F_{1}(x, y)=F(x,-y)$ is $H_{1}(x, y)=T x^{2}-T x y+V y^{2}$. Then, $H_{1}(x+y, y)=T x^{2}+T x y+V y^{2}$ is a reduced quadratic form. Consider therefore the corresponding cubic form

$$
\begin{aligned}
F_{2}(x, y)= & F(x+y,-y)=a x^{3}+(3 a-b) x^{2} y \\
& +(3 a-2 b+c) x y^{2}+(a-b+c-d) y^{3} .
\end{aligned}
$$

Suppose that $b=3 a / 2$. From $U=T$, we then have $d=-a / 4+c / 2$ so that $F_{2}(x, y)=F(x, y)$. However, it is easy to see that $F(x, y)$ would be divisible by $2 x+y$ in the ring $\mathbf{Q}[x, y]$. Therefore, $b \neq 3 a / 2$ and either $F(x, y)$ or $F_{2}(x, y)$ satisfies (ii) depending upon whether $b>3 a / 2$ or $b<3 a / 2$.

The same argument as in the preceding case shows that any form contained in $\mathscr{C}$ and satisfying (ii) must coincide with $F_{\mathscr{C}}(x, y)$ or with $F_{\mathscr{C}}(x+y,-y)$. Since the latter form does not satisfy (ii) the uniqueness of $F_{\mathscr{C}}$ follows.

Case 3. $0<U<T=V$. In this case $H(x, y)=T x^{2}+U x y+T y^{2}$. Consider the transformed cubic form

$$
\begin{aligned}
F_{1}(x, y) & =F((\operatorname{sgn} d) y,(\operatorname{sgn} d) x) \\
& =(\operatorname{sgn} d)\left(d x^{3}+c x^{2} y+b x y^{2}+a y^{3}\right)
\end{aligned}
$$

the quadratic covariant of which coincides with $H(x, y)$.

Suppose first that $d=a$. From $T=V$ it then follows that $(b-c)(3 a+b+c)=$ 0 . We cannot have $b=c$ otherwise $F(x, y)$ would be divisible by $x+y$. From $3 a+b+c=0$ we infer $U=-9 a^{2}-3 a b-b^{2}<0$ contrary to the assumption.

Suppose next that $d=-a$. From $T=V$ we get $(b+c)(3 a-b+c)=0$. We cannot have $b=-c$ otherwise $F(x, y)$ would be divisible by $x-y$. From $3 a-b+$ $c=0$ it follows that $U=9 a^{2}-3 a b+b^{2}=T$, contradicting the assumption.

Therefore, $|d| \neq a$ and either $F(x, y)$ or $F_{1}(x, y)$ satisfies (iii). The uniqueness of $F_{\mathscr{C}}(x, y)$ is proved as before.

Case 4. $0=U<T=V$. The following general identities are valid in each case

$$
\begin{aligned}
& c T-b U+3 a V=0, \\
& 3 d T-c U+b V=0 .
\end{aligned}
$$


In Case 4 we find from these identities that $c=-3 a, b=-3 d$. Therefore, $F(x, y)$ is of the form

$$
F(x, y)=a x^{3}-3 d x^{2} y-3 a x y^{2}+d y^{3} .
$$

Clearly $d \neq \pm a$ because $F(x, y)$ is irreducible in $\mathbf{Q}[x, y]$. Now the cubic forms

$$
\begin{aligned}
& F(x, y)=a x^{3}-3 d x^{2} y-3 a x y^{2}+d y^{3}, \\
& F(x,-y)=a x^{3}+3 d x^{2} y-3 a x y^{2}-d y^{3}, \\
& F((\operatorname{sgn} d) y,(\operatorname{sgn} d) x)=(\operatorname{sgn} d)\left(d x^{3}-3 a x^{2} y-3 d x y^{2}+a y^{3}\right), \\
& F(-(\operatorname{sgn} d) y,(\operatorname{sgn} d) x)=(\operatorname{sgn} d)\left(d x^{3}+3 a x^{2} y-3 d x y^{2}-a y^{3}\right),
\end{aligned}
$$

are contained in $\mathscr{C}$ and exactly one of them satisfies (iv). By changing the notation if necessary we may assume that this form is $F(x, y)$. We have thus found $F_{\mathscr{C}}(x, y)=$ $F(x, y)$ with quadratic covariant $H(x, y)=T\left(x^{2}+y^{2}\right)$.

Suppose now that $F_{1}(x, y)$ is another form in $\mathscr{C}$ satisfying (iv) and let $H_{1}(x, y)$ denote its quadratic covariant. Since $H_{1}(x, y)$ and $H(x, y)$ are equivalent reduced quadratic forms and $H(x, y)$ is improperly equivalent to itself, we must have $H_{1}(x, y)=H(x, y)$. Let $\tau$ denote a substitution with determinant $=+1$ transforming $F_{1}(x, y)$ into $F(x, y)$ or $F(x,-y)$. Then $\tau$ is an automorph of $H(x, y)$ and by $[11$, loc.cit.] $\tau$ is one of the following four substitutions:

$$
\begin{gathered}
{\left[x=x^{\prime}, y=y^{\prime}\right], \quad\left[x=-x^{\prime}, y=-y^{\prime}\right], \quad\left[x=y^{\prime}, y=-x^{\prime}\right],} \\
{\left[x=-y^{\prime}, y=x^{\prime}\right] .}
\end{gathered}
$$

Taking into account that the leading coefficients of $F_{1}(x, y)$ and $F(x, y)$ are positive, it is easy to see that $F_{1}(x, y)$ must be one of the forms (2.8). However, only one of them satisfies (iv) and therefore $F_{1}(x, y)=F(x, y)$.

Case 5. $U=T=V$. From (2.6) and (2.7) we have

$$
c-b+3 a=3 d-c+b=0
$$

implying $d=-a, c=b-3 a$. Hence, $F(x, y)$ is of the form

$$
F(x, y)=a x^{3}+b x^{2} y+(b-3 a) x y^{2}-a y^{3} .
$$

We must have $b \neq 3 a / 2$, otherwise $F(x, y)$ would be divisible by $x-y$. Further,

$$
F(-y,-x)=a x^{3}+(-b+3 a) x^{2} y-b x y^{2}-a y^{3},
$$

and either $F(x, y)$ or $F(-y,-x)$ satisfies (v), i.e., is the required $F_{\mathscr{C}}(x, y)$. Choose the notation so that this form is $F(x, y)$. Its quadratic covariant is $H(x, y)=$ $T\left(x^{2}+x y+y^{2}\right)$. Suppose that $F_{1}(x, y)$ is another form in $\mathscr{C}$ satisfying (v). As in Case 4, we find that the quadratic covariant of $F_{1}(x, y)$ must coincide with $H(x, y)$. Again let $\tau$ denote a substitution of determinant $=+1$ transforming $F_{1}(x, y)$ into $F(x, y)$ or $F(-y,-x)$. By $[11$, loc.cit.] $\tau$ must be one of the following six substitutions:

$$
\begin{aligned}
& {\left[x=x^{\prime}, y=y^{\prime}\right], \quad\left[x=-x^{\prime}-y^{\prime}, y=x^{\prime}\right], \quad\left[x=y^{\prime}, y=-x^{\prime}-y^{\prime}\right],} \\
& {\left[x=-x^{\prime}, y=-y^{\prime}\right], \quad\left[x=x^{\prime}+y^{\prime}, y=-x^{\prime}\right], \quad\left[x=-y^{\prime}, y=x^{\prime}+y^{\prime}\right] .}
\end{aligned}
$$

However, simple computations show that for such $\tau$ the substitution $\tau^{-1}$ transforms $F(x, y)$ and $F(-y,-x)$ into themselves or into identically opposite forms. Since only one of these four forms satisfies (v), we have $F_{1}(x, y)=F(x, y)$. 
3. Inequalities Concerning Reduced Cubic Forms. A table of minimal polynomials was produced by first tabulating the relevant cubic forms. For that purpose precise estimates for the coefficients of these forms in terms of the discriminant are valuable. In the following theorem we shall present a collection of such estimates all of which are best possible. The first four are classical [3], [14], but for the convenience of the reader we give a complete proof. In a less accurate form these results are contained in [6, p. 185, Lemma 1].

THEOREM 2. Let $F(x, y)=a x^{3}+b x^{2} y+c x y^{2}+d y^{3}$ be a reduced binary cubic form with discriminant $D=D(F)$. Then the following estimates are valid:

$$
\begin{gathered}
0<a<2 D^{1 / 4} / \sqrt{27}, \quad|b| \leqslant 2 D^{1 / 4} / \sqrt{3}, \\
|a d|<4 D^{1 / 2} / 27, \quad|b c|<D^{1 / 2}, \\
\max \left\{\left|a c^{3}\right|,\left|b^{3} d\right|\right\}<(35+13 \sqrt{13}) D / 216 .
\end{gathered}
$$

Remark. The example $a=1, b=6, c=3, d=-1$ shows that equality can occur in (3.1).

To show that the first inequalities in (3.1) and (3.2) are best possible take $a=4 m-2, b=6 m, c=-6 m, d=-4 m+1$, where $m$ is a (large) positive integer. Then $F(x, y)$ is clearly primitive and it is irreducible in $\mathbf{Q}[x, y]$ by Eisenstein's criterion. We have

$$
T=108 m^{2}-36 m, \quad U=108 m^{2}-108 m+18, \quad V=108 m^{2}-18 m
$$

so that $0<U<T<V$ and $F(x, y)$ is reduced. Since $D^{1 / 2}=108 m^{2}+O(m)$ we have $a D^{-1 / 4}=2 / \sqrt{27}+O\left(m^{-1}\right),|a d| D^{-1 / 2}=4 / 27+O\left(m^{-1}\right)$.

Next, take $a=1, b=2 m+4, c=2 m, d=-2$, where again $m$ is a (large) positive integer. Then,

$$
T=4 m^{2}+10 m+16, \quad U=4 m^{2}+8 m+18, \quad V=4 m^{2}+12 m+24,
$$

whence $F(x, y)$ is reduced. We have $D^{1 / 2}=4 m^{2}+O(m)$ and $b c D^{-1 / 2}=1+$ $O\left(\mathrm{~m}^{-1}\right)$ so that the second inequality (3.2) is best possible.

The fact that (3.3) is best possible will be obvious from the subsequent proof.

Proof of Theorem 2. The following general identities are easily seen to be true:

$$
\begin{aligned}
(2 b T-3 a U)^{2}+27 a^{2} D & =4 T^{3}, \\
(2 c T-b U)^{2}+3 b^{2} D & =4 T^{2} V, \\
(2 b V-c U)^{2}+3 c^{2} D & =4 T V^{2}, \\
(2 c V-3 d U)^{2}+27 d^{2} D & =4 V^{3} .
\end{aligned}
$$

Since the form is reduced, we have

$$
D=\left(4 T V-U^{2}\right) / 3 \geqslant T V \geqslant T^{2} .
$$

From (3.4), (3.5), (3.8) we immediately obtain (3.1) with the reservation that the upper estimate of $a$ might not be a strict inequality. However, in the case of equality we must also have equality in (3.8) and $2 b T-3 a U=0$. This would imply $T=U=$ $V$ and $2 b=3 a$ contrary to the proof of Theorem 1, Case 5 .

On combining (3.4), (3.7), (3.8), we have

$$
(27 a d D)^{2} \leqslant 16(T V)^{3} \leqslant 16 D^{3}
$$

implying $\mid$ ad $\mid<4 D^{1 / 2} / 27$ because equality cannot occur. 
Using the identity

$$
b^{2} V-b c U+c^{2} T=T V
$$

we find

$$
2(T V)^{1 / 2}|b c| \leqslant b^{2} V+c^{2} T=T V+b c U \leqslant T V+|b c U|,
$$

whence, by (3.8),

$$
\begin{aligned}
3 D|b c| & =\left(2(T V)^{1 / 2}+|U|\right)\left(2(T V)^{1 / 2}-|U|\right)|b c| \\
& \leqslant\left(2(T V)^{1 / 2}+|U|\right) T V \leqslant 3(T V)^{3 / 2} \leqslant 3 D^{3 / 2}
\end{aligned}
$$

so that $|b c| \leqslant D^{1 / 2}$. If equality occurs then $T=U=V$ and $b=c$. But in the proof of Theorem 1, Case 5 we saw that $c=b-3 a$, a contradiction. We have thus proved (3.2) and it remains to prove (3.3).

Put $\kappa=(35+13 \sqrt{13}) / 216$. We shall show first that $\left|a c^{3}\right|<\kappa D$. From (2.4) and (3.4) we find

$$
a c^{3} / D=\left(b^{2}-T\right)^{3} /\left(4 T^{3}-(2 b T-3 a U)^{2}\right) .
$$

We have the identity

$$
9 a^{2} V-3 a b U+b^{2} T=T^{2} .
$$

Put

$$
x=3 a V^{1 / 2} T^{-1}, \quad y=3 a U T^{-3 / 2}, \quad z=2 b T^{-1 / 2}-3 a U T^{-3 / 2} .
$$

From (3.11) and from the conditions of reduction we get

$$
x>0, \quad|y| \leqslant x, \quad 4 x^{2}-y^{2}+z^{2}=4
$$

and the right-hand side of (3.10) takes the form

$$
\varphi(x, y, z)=\left((y+z)^{2}-4\right)^{3} /\left(64\left(4 x^{2}-y^{2}\right)\right) .
$$

We have to study the function $\varphi(x, y, z)$ subject to the constraints (3.12). We may suppose that $z \geqslant 0$ because the change of the signs of $y$ and $z$ leaves $\varphi$ and (3.12) unaltered. It follows, in particular, from (3.12) that $3 x^{2}+z^{2} \leqslant 4$, and thus

$$
|y| \leqslant x \leqslant 2 / \sqrt{3}, \quad 0 \leqslant z<2 .
$$

If $(x, y, z) \rightarrow\left(0, y_{0}, z_{0}\right)$ subject to (3.12) and (3.13), then $y_{0}=0, z_{0}=2$, and it is easy to see that $\lim \varphi(x, y, z)=0$. We may therefore assume that a point $(x, y, z)$ satisfying (3.12) and (3.13) is so chosen that the function $|\varphi|$ subject to these constraints attains its maximum at that point.

Suppose first that $|y+z| \geqslant 2$. From (3.13) we have $y>0$. Since

$$
|\varphi(x, y, z)|=\left(\left(y+\left(4-4 x^{2}+y^{2}\right)^{1 / 2}\right)^{2}-4\right)^{3} /\left(64\left(4 x^{2}-y^{2}\right)\right)
$$

is an increasing function of $y$ we must have $y=x$ so that

$$
\begin{aligned}
|\varphi(x, y, z)| & =\left(\left(x+\left(4-3 x^{2}\right)^{1 / 2}\right)^{2}-4\right)^{3} /\left(192 x^{2}\right) \\
& =\left(x\left(4-3 x^{2}\right)^{1 / 2}-3 x^{2}+2 x^{4}\right) / 6 .
\end{aligned}
$$


Since $x+\left(4-3 x^{2}\right)^{1 / 2}=y+z \geqslant 2$ implies $x \leqslant 1$, we have to compute the maximum of the function (3.14) in the interval $(0,1]$. This is a trivial task, the maximum is $(-35+13 \sqrt{13}) / 216$ attained at the point $x=((5-\sqrt{13}) / 12)^{1 / 2}$.

Suppose next that $|y+z|<2$. For $z \leqslant 1$ we have

$$
\begin{aligned}
|\varphi(x, y, z)| & =\left(4-(y+z)^{2}\right)^{3} /\left(64\left(4-z^{2}\right)\right) \\
& \leqslant 1 /\left(4-z^{2}\right) \leqslant 1 / 3<\kappa .
\end{aligned}
$$

Suppose therefore that $z>1$. Then (3.12) implies $|y| \leqslant x<1$ so that $0<y+z<2$. From the choice of the point $(x, y, z)$ it now follows that we must have $y=-x$; otherwise we could either change the sign of $y$ or diminish $y$ slightly and change $x$ correspondingly in order to keep $z$ fixed, which would give us a larger value of $|\varphi(x, y, z)|$. Therefore,

$$
\begin{aligned}
|\varphi(x, y, z)| & =\left(4-\left(-x+\left(4-3 x^{2}\right)^{1 / 2}\right)^{2}\right)^{3} /\left(192 x^{2}\right) \\
& =\left(x\left(4-3 x^{2}\right)^{1 / 2}+3 x^{2}-2 x^{4}\right) / 6
\end{aligned}
$$

and on computing the maximum value of the function $(3.15)$ in the interval $(0,1)$ we obtain $|\varphi(x, y, z)|=\kappa$ for

$$
(x, y, z)=\left(((5+\sqrt{13}) / 12)^{1 / 2},-((5+\sqrt{13}) / 12)^{1 / 2},((11-\sqrt{13}) / 4)^{1 / 2}\right) .
$$

Hence $\left|a c^{3}\right|<\kappa D$ as asserted.

The proof of $\left|b^{3} d\right|<\kappa D$ is similar. We start from the expression

$$
b^{3} d / D=\left(c^{2}-V\right)^{3} /\left(4 V^{3}-(2 c V-3 d U)^{2}\right)
$$

which is a consequence of (2.4) and (3.7), and use the identity

$$
9 d^{2} T-3 c d U+c^{2} V=V^{2} .
$$

This time we write

$$
x=3|d| T^{1 / 2} V^{-1}, \quad y=3 d U V^{-3 / 2}, \quad z=2 c V^{-1 / 2}-3 d U V^{-3 / 2},
$$

and the proof proceeds exactly as before.

4. Normalized Cubic Polynomial. Let $F(x, y)=a x^{3}+b x^{2} y+c x y^{2}+d y^{3}$ be a reduced cubic form of discriminant $D=D(F)$. The polynomial $f(x)$ associated with the form $F(x, y)$ is defined as

$$
f(x)=a^{-1} F(x+(e b-s) / 3,-e a),
$$

where

$$
s=\left\{\begin{array}{ll}
1 & \text { if } b \neq 0 \bmod 3, \\
0 & \text { if } b \equiv 0 \bmod 3 ;
\end{array} \quad e=\left\{\begin{aligned}
1 & \text { if } b \equiv 1 \bmod 3 \\
-1 & \text { if } b \neq 1 \bmod 3
\end{aligned}\right.\right.
$$

Clearly $f(x)$ is a monic cubic polynomial with integral coefficients in which $-s$ is the coefficient of $x^{2}$. Writing

$$
f(x)=x^{3}-s x^{2}+q x-n,
$$


we have

$$
\begin{gathered}
q=a c-\left(b^{2}-s\right) / 3=(s-T) / 3, \\
n=(2 e b+s)(e b-s)^{2} / 27-a c(e b-s) / 3+e a^{2} d
\end{gathered}
$$

since $s^{2}=s$ and $e^{2}=1$. The discriminant of the polynomial $f(x)$ is

$$
\begin{aligned}
s q^{2}-4 q^{3}-4 s n-27 n^{2}+18 s q n & =a^{2} D \\
= & 4 T^{3} / 27-3(3 n-s q+2 s / 9)^{2} .
\end{aligned}
$$

We note that the reduced form $F(x, y)$ is not uniquely determined by its associated polynomial. This is seen trivially by considering the forms $a x^{3}+c x y^{2}+$ $d y^{3}$ and $x^{3}+a c x y^{2}+a^{2} d y^{3}$ where $c<-3 a<-3,0<d<-c /(3 a)$, and $\operatorname{gcd}(a, c, d)=1$. (In order to ensure the required irreducibility assume, e.g., that there exists a prime $p$ such that $p+a, p|c, p| d, p^{2}+d$.) Then the forms are reduced and they both have associated polynomial $x^{3}+a c x+a^{2} d$.

It is more difficult to find examples of a pair of reduced cubic forms both having the same leading coefficient and the same associated polynomial. One such pair is

$$
\begin{gathered}
k^{3} x^{3}-3 k^{2}(k+1) x y^{2}+\left(k^{3}+k^{2}-3\right) y^{3}, \\
k^{3} x^{3}+3 k^{2}(k-1) x^{2} y-3 k(3 k-1) x y^{2}-\left(k^{3}+2 k^{2}-6 k+4\right) y^{3},
\end{gathered}
$$

where $k$ is a positive integer $\equiv-1 \bmod 18$. Both forms have associated polynomial

$$
x^{3}-3 k^{5}(k+1) x+k^{8}(k+1)-3 k^{6} \text {. }
$$

Suppose now that $K$ is a totally real cubic field of discriminant $D$ and let $\mathscr{B}=\{1, \alpha, \omega\}$ be an integral basis for $\mathcal{O}$ containing 1 (called unitary in $[9$, Section 15]). Following Davenport and Heilbronn [7], [8] we assign to $\mathscr{B}$ the cubic form

$$
\begin{aligned}
F(x, y ; \mathscr{B})= & D^{-1 / 2}\left(\left(\alpha^{\prime}-\alpha^{\prime \prime}\right) x+\left(\omega^{\prime}-\omega^{\prime \prime}\right) y\right)\left(\left(\alpha^{\prime \prime}-\alpha\right) x+\left(\omega^{\prime \prime}-\omega\right) y\right) \\
& \times\left(\left(\alpha-\alpha^{\prime}\right) x+\left(\omega-\omega^{\prime}\right) y\right) .
\end{aligned}
$$

It is easily seen that the equivalence class containing the form $F(x, y ; \mathscr{B})$ is independent of the choice of $\mathscr{B}$ and thus depends only on $K$. We denote this class by $\mathscr{C}(K)$. By an abuse of notation write $F_{K}(x, y)=F_{\mathscr{C}(K)}(x, y)$ for the reduced form in the class $\mathscr{C}(K)$ and let $\mathscr{R}$ be the set of all forms $F_{K}(x, y), K$ ranging over the set of all totally real cubic fields. From [8, p. 418, Proposition 4] we have

THEOREM 3. The assignment $K \mapsto F_{K}(x, y)$ induces a bijective discriminant-preserving map of the set of triplets of conjugate totally real cubic fields onto $\mathscr{R}$.

Here, of course, the triplet is coalescent if $K$ is cyclic. From [8] it is easy to derive the following result which is fundamental in the search of all totally real cubic fields with discriminants in a given range.

THEOREM 4. Let $F(x, y)=a x^{3}+b x^{2} y+c x y^{2}+d y^{3}$ be a reduced cubic form of discriminant $D$. We have $F(x, y) \in \mathscr{R}$ if and only if the following Davenport-Heilbronn conditions are satisfied:

(DH2) $D \equiv 0 \bmod 16$; if $D \equiv 4 \bmod 16$ then $b \equiv c \bmod 2$.

(DH3) If $D \equiv 0 \bmod 9$ then $b \equiv c \equiv 0 \bmod 3$ and $a d\left(a^{2}-d^{2}-a c+b d\right) \equiv 3$ or $6 \bmod 9$.

(DHp) For every prime $p \geqslant 5$ such that $D \equiv 0 \bmod p^{2}$ we have $D \neq 00 \bmod p^{3}$, $a \not \equiv 0 \bmod p^{2}, T=b^{2}-3 a c \equiv 0 \bmod p$. 
Proof. Prime $p=2$. By $[8$, p. 407] the local condition at the prime 2 is equivalent to the foliowing one: If $D \equiv 0$ or $4 \bmod 16$, then $F(x, y) \equiv(a x+d y)^{3} \bmod 2$, and the congruence $F(x, y) \equiv 2 \bmod 4$ has a solution.

Suppose first that this condition holds. Clearly $b \equiv c \equiv a d \bmod 2$. If $a d$ is even we may suppose, e.g., that $a$ is even and $d$ is odd. The solution of $F(x, y) \equiv 2 \bmod 4$ must be $(x, y)=(1,0)$ whence $a \equiv 2 \bmod 4$ and $D \equiv a^{2} d^{2} \equiv 4 \bmod 16$. If $a d$ is odd apply first the substitution $x=x^{\prime}+y^{\prime}, y=y^{\prime}$. Hence, (DH2) holds.

Suppose conversely that $(\mathrm{DH} 2)$ is true. Let $D \equiv 4 \bmod 16$, otherwise there is nothing to prove. If $b \equiv c \equiv 0 \bmod 2$ we see from (2.2) that $D \equiv 5 a^{2} d^{2}+$ $2 a b c d \bmod 16$. Thus $a d$ is even and $D \equiv a^{2} d^{2} \bmod 16$ (e.g., let $a$ be even and $d$ odd). Since $D \not \equiv 0 \bmod 16$ we have $a \equiv 2 \bmod 4$ so that $F(x, y) \equiv y^{3} \bmod 2, F(1,0) \equiv$ $2 \bmod 4$. If $b \equiv c \equiv 1 \bmod 2$ we get from (2.2) that $a d$ is odd. Apply the same substitution as above.

Prime $p=3$. The local condition in [8] at the prime 3 can be written as follows: If $D \equiv 0 \bmod 9$ then $F(x, y) \equiv(a x+d y)^{3} \bmod 3$, and the congruence $F(x, y) \equiv$ $3 e \bmod 9$ has a solution for $e=1$ or 2 .

Suppose first that this condition holds and that $D \equiv 0 \bmod 9$. Clearly $b \equiv c \equiv$ $0 \bmod 3$. If $3 \mid a$ the solvability of $F(x, y) \equiv 3 e \bmod 9$ plainly implies $9+a$, whence $a d\left(a^{2}-d^{2}-a c+b d\right) \equiv-a d^{3} \equiv 3$ or $6 \bmod 9$. The same conclusion holds if $3 \mid d$. Therefore, let $a d \neq \equiv 0 \bmod 3$. Then $a^{2}-d^{2}-a c+b d \equiv 0 \bmod 3$. We may assume that the solution of $F(x, y) \equiv 3 e \bmod 9$ is $(x, y)=(1,1)$ or $(1,-1)$. Accordingly either $a+b+c+d$ or $a-b+c-d$ is $\equiv 3$ or $6 \bmod 9$. In the first case, we have $a+d \equiv 0 \bmod 3$ and

$$
\begin{aligned}
a^{2}-d^{2}-a c+b d & \equiv a^{2}-d^{2}+(a-d) c+(a-d) b \\
& \equiv(a-d)(a+b+c+d) \equiv 3 \text { or } 6 \bmod 9
\end{aligned}
$$

and similarly, in the other case. Hence (DH3) is true.

Suppose conversely that $(\mathrm{DH} 3)$ holds and $D \equiv 0 \bmod 9$. From $b \equiv c \equiv 0 \bmod 3$ we immediately have $F(x, y) \equiv(a x+d y)^{3} \bmod 3$. If $3 \mid a$ then $9+a$ by (DH3), so that $F(1,0) \equiv 3$ or $6 \bmod 9$. If $3 \mid d$, we similarly have $F(0,1) \equiv 3$ or $6 \bmod 9$. Let $a d \equiv 0 \bmod 3$. From $(\mathrm{DH} 3),(a+d)(a-d) \equiv 0 \bmod 3$, and one may reverse the argument above.

Prime $p \geqslant 5$. The condition from [8] is the following one: If $D \equiv 0 \bmod p^{2}$ then $F(x, y) \equiv r(h x+k y)^{3} \bmod p$ for some integers $r, h, k$, and the congruence $F(x, y)$ $\equiv e p \bmod p^{2}$ has a solution for some $e \not \equiv 0 \bmod p$.

Suppose first that this condition is true and $D \equiv 0 \bmod p^{2}$. From $[8$, p. 410 , Lemma 6] we have $D \equiv 0 \bmod p^{3}$. Further $T \equiv\left(3 r h^{2} k\right)^{2}-3 r h^{3} 3 r h k^{2} \equiv 0 \bmod p$, and similarly $U \equiv V \equiv 0 \bmod p$. If $p^{2} \mid a$, then from $T \equiv V \equiv 0 \bmod p$ we would have $b \equiv c \equiv 0 \bmod p$ and from $(2.2), D \equiv 0 \bmod p^{3}$, a contradiction. Thus (DH $\left.p\right)$ holds.

Suppose conversely that $(\mathrm{DH} p)$ is true, and that $D \equiv 0 \bmod p^{2}$. If $p+a$, we find from $T \equiv U \equiv 0 \bmod p$ that $c \equiv b^{2} /(3 a) \bmod p$ and $d \equiv b^{3} /\left(27 a^{2}\right) \bmod p$, and so $F(x, y) \equiv(3 a x+b y)^{3} /\left(27 a^{2}\right) \bmod p$. If $p \mid a$ it follows from $T \equiv 0 \bmod p$ that $p \mid b$ and hence $D \equiv-4 a c^{3} \bmod p^{2}$ by (2.2). Since $p^{2}+a$ we get $p \mid c$ and thus $F(x, y) \equiv$ $d y^{3} \bmod p$. The rest now follows from [8, p. 410, Lemma 6]. 
A polynomial $f(x)$ is said to be a normalized cubic polynomial (abbreviated NCP) iff there is a totally real cubic field $K$ such that $f(x)$ is the polynomial associated with the form $F_{K}(x, y)$. Obviously $K=\mathbf{Q}(\alpha)$, where $f(\alpha)=0$. We shall call $\alpha$ a normalized primitive element (abbreviated NPE) of $K$ over $\mathbf{Q}$. Thus, every noncyclic $K$ has a unique naturally defined NPE, whereas a cyclic $K$ contains a triplet of such elements.

5. Properties of Normalized Cubic Polynomials. In the following theorem we shall prove first that for an NPE, the conjugates have least standard deviation among all irrational algebraic integers of the cubic field in question. Later on in this section we shall see that this property almost characterizes an NPE of given trace. In the foregoing section we saw that two reduced cubic forms may have the same leading coefficient and the same associated polynomial. Here, we shall see that this is not possible if the polynomial is an NCP. We shall also construct an algorithm by means of which the corresponding form in $\mathscr{R}$ can be computed when the polynomial is given.

THEOREM 5. Let $\alpha$ be an NPE of a totally real cubic field $K$ and put $\operatorname{Irr}(\alpha, \mathbf{Q})=f(x)$ $=x^{3}-s x^{2}+q x-n$. For any $\xi \in K$, write

$$
R(\xi)=\frac{1}{2}\left(\left(\xi-\xi^{\prime}\right)^{2}+\left(\xi^{\prime}-\xi^{\prime \prime}\right)^{2}+\left(\xi^{\prime \prime}-\xi\right)^{2}\right) .
$$

(i) We have $R(\alpha)=s-3 q=\min \{R(\xi)\}$, the minimum being taken over all irrational algebraic integers $\xi$ of $K$.

(ii) For any given positive integer $k$, there exists at most one reduced cubic form $F(x, y)$ with leading coefficient $k$, such that $f(x)$ is the polynomial associated with $F(x, y)$.

(iii) Denote $F_{K}(x, y)=a x^{3}+b x^{2} y+c x y^{2}+d y^{3} \in \mathscr{R}$. The form $F_{K}(x, y)$ can be traced back from the polynomial $f(x)$ by means of the following algorithm.

(I) The leading coefficient $a$ is the largest positive integer for which an integer $t$ can be found such that

$$
f(t) \equiv 0 \bmod a^{2}, \quad f^{\prime}(t) \equiv 0 \bmod a .
$$

We have $b^{2}-3 a c=T=s-3 q=R(\alpha)$.

(II) If $s=0$, then

$$
\begin{aligned}
& b=3 t+3 a\left[\frac{1}{2}-t / a-9 n /(2 a T)\right], \\
& c=\left(b^{2}-T\right) /(3 a), \\
& d=\left(b^{3}-3 b T-27 n\right) /\left(27 a^{2}\right) .
\end{aligned}
$$

(III) If $s=1$, determine first

$$
\begin{aligned}
& g=\frac{1}{2}-t / a-(T-1) /(6 a T)-9 n /(2 a T), \\
& b_{*}=3 t-1+3 a[g] .
\end{aligned}
$$

(III.1) If $g \in \mathbf{Z}$, then

$$
\begin{aligned}
& b=\max \left\{b_{*}, 3 a-b_{*}\right\}, \\
& c=\left(b^{2}-T\right) /(3 a) \\
& d=(b c-T) /(9 a) .
\end{aligned}
$$


(III.2) If $g \notin \mathbf{Z}$, then writing

$$
d_{*}=\left(b_{*}^{3}+1-3\left(b_{*}+1\right) T-27 n\right) /\left(27 a^{2}\right),
$$

we have $c=\left(b_{*}^{2}-T\right) /(3 a),(b, d)=\left(b_{*}, d_{*}\right)$ or $\left(-b_{*},-d_{*}\right)$, chosen so that the condition (i) or (iii) in Theorem 1 is satisfied.

Proof. (i) By definition, $f(x)$ is the polynomial associated with the form $F_{K}(x, y)$. From (4.4), $T=b^{2}-3 a c=s-3 q$, and on the other hand, clearly, $R(\alpha)=s^{2}-3 q$ $=s-3 q$. Let $H(x, y)$ denote the quadratic covariant of $F_{K}(x, y)$. Choose an irrational algebraic integer $\beta$ of $K$ so that $R(\beta)$ is least possible. By the minimality of $R(\beta),(\beta-h) / k$ cannot be an algebraic integer for any pair of rational integers $h, k$ with $k>1$. It follows that $\mathcal{O}$ has an integral basis $\mathscr{B}$ of the form $\{1, \beta$, $\left.\left(u+v \beta+\beta^{2}\right) / w\right\}$ for some integers $u, v, w$ with $w>0$. Since $w=[\mathcal{O}: \mathbf{Z}[\beta]]$, we find that $w$ is equal to the leading coefficient $\left(\beta^{\prime}-\beta^{\prime \prime}\right)\left(\beta^{\prime \prime}-\beta\right)\left(\beta-\beta^{\prime}\right) D^{-1 / 2}$ of the form $F(x, y ; \mathscr{B})$, provided that the order of the conjugates of $\beta$ is suitably chosen. Here, $D=D\left(F_{K}\right)$ denotes the discriminant of $K$. Therefore,

$$
\begin{aligned}
F(x, y ; \mathscr{B}) & =w(x+\gamma y)\left(x+\gamma^{\prime} y\right)\left(x+\gamma^{\prime \prime} y\right), \\
\gamma & =(v+\operatorname{Tr}(\beta)-\beta) / w .
\end{aligned}
$$

A simple computation now shows that the first quantity (2.4) for the form $F(x, y ; \mathscr{B})$ equals $R(\beta)$. Since $F(x, y ; \mathscr{B})$ and $F_{K}(x, y)$ are equivalent, so are their quadratic covariants, and therefore $R(\beta)$, being the leading coefficient of the former covariant, is an integer represented by the reduced quadratic form $H(x, y)$. Since $T$ is the least nonzero integer represented by $H(x, y), R(\beta) \geqslant T$, and thus necessarily $R(\beta)=T$.

(ii) Let $F(x, y)$ be a reduced cubic form with leading coefficient $k$ such that $f(x)$ is the polynomial associated with $F(x, y)$. By $(4.1), f(x)=k^{-1} F(x-t, \pm k)$ for some integer $t$. Hence

$$
f(t) \equiv 0 \bmod k^{2}, \quad f^{\prime}(t) \equiv 0 \bmod k .
$$

It is easy to see that an integer $t$ satisfying (5.3) is unique mod $k$. Suppose, indeed, that $t^{\prime}$ is another such integer. It follows from a theorem of Voronoi [9, p.111, Theorem I] that the numbers

$$
\left(u^{2}-s u+q+(u-s) \alpha+\alpha^{2}\right) / k, \quad u=t \text { or } t^{\prime}
$$

are algebraic integers. Their difference is $\left(t-t^{\prime}\right)\left(t+t^{\prime}-s+\alpha\right) / k$. However, as was seen above, a number of this type can be integral only for $t-t^{\prime} \equiv 0 \bmod k$. The argument in the subsequent proof of (iii) now gives the uniqueness of $F(x, y)$. We observe that (ii) is true under the weaker assumption that no number of the form $(\alpha-h) / k$ with $h, k \in \mathbf{Z}, k>1$ is an algebraic integer, which is equivalent to the fact that there exists an integral basis for $\mathcal{O}$ containing 1 and $\alpha$.

(iii) Since the discriminant of the polynomial $f(x)$ equals $a^{2} D$, it follows immediately from the theorem of Voronoi cited above that (I) is true. From the foregoing proof of (ii) we have $t \equiv(s-e b) / 3 \bmod a$.

Consider first the case $s=0$. Then $e=-1$ and $t \equiv b / 3 \bmod a$. Putting $b / 3=t+$ $r a$, we have from (4.5)

$$
a U=a b c-9 a^{2} d=2(t+r a) T+9 n .
$$

Since $-T<U \leqslant T$, we infer

$$
-a T<2(t+r a) T+9 n \leqslant a T,
$$


so that $r=\left[\frac{1}{2}-t / a-9 n /(2 a T)\right]$, which gives the value of $b$ in (II). The rest of (II) is clearly true, the expression for $d$ being a consequence of (4.5).

Suppose now that $s=1$. In this case the argument is slightly more complicated because we do not know beforehand whether $b \equiv 1$ or $-1 \bmod 3$. Writing $(1-e b) / 3$ $=t+r a$, we have this time from (4.5)

$$
-e a U=2(t+r a) T+(T-1) / 3+9 n,
$$

so that

$$
r=-e U /(2 T)-t / a-(T-1) /(6 a T)-9 n /(2 a T) .
$$

It follows from the inequality $-T<U \leqslant T$ that $g-1 \leqslant r \leqslant g$.

If $g \in \mathbf{Z}$ then $r=g$ or $g-1$, which is possible only for $U=T$. If $b \equiv-1 \bmod 3$, then $e=-1$ and $r=g$ so that $(1+b) / 3=t+r a$ implies $b=b_{*}$. If $b \equiv 1 \bmod 3$, then $e=1$ and $r=g-1$ so that $(1-b) / 3=t+r a$ implies $b=3 a-b_{*}$. On the other hand, we have $b>3 a / 2$ by Theorem 1 , (ii) and (v), and therefore $b=$ $\max \left\{b_{*}, 3 a-b_{*}\right\}$ in both cases. The assertion (III.1) now follows, the value of $d$ being obtained from the equation $U=T$.

If $g \notin \mathbf{Z}$, then $r=[g],-e b=b_{*}$, and from (4.5), $-e d=d_{*}$. Clearly (III.2) is true.

The practical implementation of the algorithm in order to compute $F_{K}(x, y)$ is facilitated by the fact that the computer listings contain the number $a$ and the residue class of $t-s \bmod a$. For future reference we record here the obvious equality

$$
a=[\mathcal{O}: \mathbf{Z}[\alpha]]
$$

THEOREM 6. Let $K$ be a given totally real cubic field. Put $F_{K}(x, y)=a x^{3}+b x^{2} y+$ $c x y^{2}+d y^{3}$ and let $H(x, y)=T x^{2}+U x y+V y^{2}$ denote the quadratic covariant of $F_{K}(x, y)$. Then $K$ is cyclic over $\mathbf{Q}$ if and only if $T=U=V$. If this is the case, then $c=b-3 a, d=-a$, the conductor of $K$ is equal to $T$, and we have

$$
T=\left(u^{2}+3 v^{2}\right) / 4
$$

for $u= \pm(2 b-3 a), v=3 a$. Assuming that the sign of $u$ is suitably chosen, $u$ and $v$ satisfy the conditions

$$
\begin{array}{ll}
u \equiv 2 \bmod 3, v \equiv 0 \bmod 3, v>0 & \text { for } 3+T, \\
u \equiv 6 \bmod 9, v \equiv 3 \operatorname{or} 6 \bmod 9, v>0 & \text { for } 3 \mid T
\end{array}
$$

introduced by Hasse [12, p. 12]. The set $\left\{\alpha, \alpha^{\prime}, \alpha^{\prime \prime}\right\}$ of the NPE's of K coincides, apart from sign, with the set of the Gaussian periods for a generating cubic character of $K$.

Proof. If $T=U=V$, then by (2.5) the discriminant of $K$ equals $T^{2}$ so that $K$ is cyclic over $\mathbf{Q}$ with conductor $T$. Suppose therefore, conversely for the rest of the proof, that $K$ is cyclic over $\mathbf{Q}$. From the results of Hasse, it follows that we can write the conductor $T$ of $K$ in the form (5.5), where $u$ and $v$ satisfy (5.6). Take $a=v / 3$, $b=v / 2+|u| / 2$ and consider the form

$$
F(x, y)=a x^{3}+b x^{2} y+(b-3 a) x y^{2}-a y^{3} .
$$

It is well-known that either $T$ or $T / 9$ is a square-free integer. Since $T=9 a^{2}-3 a b$ $+b^{2}$ and $3+\operatorname{gcd}(a, b)$ by $(5.6)$, it follows that $\operatorname{gcd}(a, b)=1$ so that $F(x, y)$ is primitive. We have

$$
a^{-1} F(3 x-b y, 3 a y)=27 x^{3}-9 T x y^{2}+|u| T y^{3},
$$


so that $F(x, y)$ is irreducible in the ring $\mathbf{Q}[x, y]$ by Eisenstein's criterion if $T \neq 9$. The same is clearly also true for $T=9$. The quadratic covariant of $F(x, y)$ is $T\left(x^{2}+x y+y^{2}\right)$, whence $F(x, y)$ is reduced. It is easy to see that the DH-conditions in Theorem 4 are satisfied so that $F(x, y) \in \mathscr{R}$. Using the notation (4.2) the polynomial associated with the form $F(x, y)$ is

$$
\begin{aligned}
f(x) & =x^{3}-s x^{2}+((s-T) / 3) x-(e(2 b-3 a) T-3 s T+s) / 27 \\
& = \begin{cases}x^{3}-x^{2}+((1-T) / 3) x-(T(u-3)+1) / 27 & \text { if } 3+T, \\
x^{3}-(T / 3) x+|u| T / 27 & \text { if } 3 \mid T .\end{cases}
\end{aligned}
$$

Let $\boldsymbol{\theta}, \boldsymbol{\theta}^{\prime}, \boldsymbol{\theta}^{\prime \prime}$ denote the Gaussian periods for a generating character of $K$ multiplied by \pm 1 as in [16, p. 7] and let $\alpha, \alpha^{\prime}, \alpha^{\prime \prime}$ denote the zeros of $f(x)$. Now, comparing $f(x)$ with the minimal polynomial of $\theta\left[16\right.$, pp. 8-9], we see that $\alpha= \pm \theta^{(i)}$ for some $i$. Therefore, $F(x, y)$ must be $F_{K}(x, y)$, i.e., the image of $K$ under the bijective Davenport-Heilbronn mapping.

THEOREM 7. Let $\alpha$ be an NPE of a totally real cubic field $K, f(x)=\operatorname{Irr}(\alpha, \mathbf{Q})=x^{3}$ $-s x^{2}+q x-n$ the polynomial associated with $F_{K}(x, y)=a x^{3}+b x^{2} y+c x y^{2}+$ $d y^{3}$, and let $H(x, y)=T x^{2}+U x y+V y^{2}$ denote the quadratic covariant of $F_{K}(x, y)$. For any $\beta \in \mathcal{O}$ we have $R(\beta)=R(\alpha)$ if and only if $\beta$ is of the following form:

(i) If $T<V, \beta= \pm \alpha+h$, where $h \in \mathbf{Z}$.

(ii) If $U \neq T=V$, either $\beta= \pm \alpha+h$ or

$$
\beta= \pm\left(t^{2}-s t+q+(t-s) \alpha+\alpha^{2}\right) / a+h,
$$

where $h \in \mathbf{Z}$ and $t=(s-e b) / 3$, e being defined by (4.2).

(iii) If $U=T=V, \beta= \pm \alpha^{(i)}+h$, where $i \in\{0,1,2\}$ and $h \in \mathbf{Z}$.

Proof. It is easy to check that if $\beta$ is of one of the particular types in the theorem, then $\beta \in \mathcal{O}$ and $R(\beta)=R(\alpha)$. Suppose, therefore conversely, that $\beta \in \mathcal{O}$ and $R(\beta)=R(\alpha)$. Denote $\operatorname{Irr}(\beta, \mathbf{Q})=f_{1}(x)=x^{3}-s_{1} x^{2}+q_{1} x-n_{1}$. As in the proof of Theorem 5 we conclude from the minimality of $R(\beta)$, using Voronoi's theorem, that $\mathcal{O}$ has an integral basis of the form $\mathscr{B}=\{1, \beta, \rho\}$, where

$$
\rho=\left(t_{1}^{2}-s_{1} t_{1}+q_{1}+\left(t_{1}-s_{1}\right) \beta+\beta^{2}\right) / a_{1} .
$$

Here $t_{1}$ is determined mod $a_{1}$ by

$$
f_{1}\left(t_{1}\right) \equiv 0 \bmod a_{1}^{2}, \quad f_{1}^{\prime}\left(t_{1}\right) \equiv 0 \bmod a_{1},
$$

and $a_{1}$ is the largest natural number for which these congruences have a solution. Denote $F_{1}(x, y)=F(x, y ; \mathscr{B})$. Arranging the conjugates of $\beta$ suitably, we have by (5.2),

$$
\begin{aligned}
F_{1}(x, y)= & a_{1}\left(x+\left(\left(t_{1}-\beta\right) / a_{1}\right) y\right)\left(x+\left(\left(t_{1}-\beta^{\prime}\right) / a_{1}\right) y\right) \\
& \times\left(x+\left(\left(t_{1}-\beta^{\prime \prime}\right) / a_{1}\right) y\right) .
\end{aligned}
$$

From (4.1) we obtain a similar factorization

$$
\begin{aligned}
F_{K}(x, y)= & a(x-(e(t-\alpha) / a) y)\left(x-\left(e\left(t-\alpha^{\prime}\right) / a\right) y\right) \\
& \times\left(x-\left(e\left(t-\alpha^{\prime \prime}\right) / a\right) y\right)
\end{aligned}
$$


where $t=(s-e b) / 3$. Let $H_{1}(x, y)$ denote the quadratic covariant of $F_{1}(x, y)$. In the proof of Theorem 5 we saw that the leading coefficient of $H_{1}(x, y)$ is $R(\beta)=$ $R(\alpha)=T$. Let $\left(h_{i j}\right)$ denote the two-by-two matrix of a unimodular integral homogeneous linear substitution transforming $F_{K}(x, y)$ into $F_{1}(x, y)$, i.e.,

$$
F_{1}(x, y)=F_{K}\left(h_{11} x+h_{12} y, h_{21} x+h_{22} y\right) \text {. }
$$

Then, correspondingly,

$$
H_{1}(x, y)=H\left(h_{11} x+h_{12} y, h_{21} x+h_{22} y\right)
$$

and, in particular,

$$
T=T h_{11}^{2}+U h_{11} h_{21}+V h_{21}^{2} .
$$

Consider first the case $T<V$. It is well-known (and easy to see) that (5.9) holds only for $h_{11}= \pm 1, h_{21}=0$. From the unimodularity of the substitution it follows that $h_{22}= \pm 1$. Since $a$ and $a_{1}$ are positive, we must have $h_{11}=1$ and $a=a_{1}$. From Theorem 6 we know that the conjugate fields of $K$ are distinct and, therefore, we conclude from the factorizations (5.7) and (5.8), that

$$
x+h_{12} y \pm((t-\alpha) / a) y=x+\left(\left(t_{1}-\beta\right) / a\right) y .
$$

Thus (i) is true.

Consider next the case $U \neq T=V$. In this case (5.9) has the additional solution $h_{11}=0, h_{21}= \pm 1$. Then $h_{12}= \pm 1$, and from (5.7) and (5.8) we obtain

$$
h_{12} y-(e(t-\alpha) / a)\left(h_{21} x+h_{22} y\right)=C\left(x+\left(\left(t_{1}-\beta\right) / a_{1}\right) y\right),
$$

where $C$ is a constant. The leading coefficient of $F_{1}(x, y)$ is

$$
a_{1}=-e h_{21}(t-\alpha)\left(t-\alpha^{\prime}\right)\left(t-\alpha^{\prime \prime}\right) / a^{2} .
$$

From (5.10) and (5.11) we have by a simple computation

$$
\begin{aligned}
\pm \beta & =\left(t-\alpha^{\prime}\right)\left(t-\alpha^{\prime \prime}\right) / a+h=\left(t^{2}-t(s-\alpha)+\alpha^{\prime} \alpha^{\prime \prime}\right) / a+h \\
& =\left(t^{2}-s t+q+(t-s) \alpha+\alpha^{2}\right) / a+h
\end{aligned}
$$

for some integer $h$. Hence (ii) holds.

Consider finally the cyclic case $U=T=V$. In this case the number $\alpha$ in the previous expressions is replaceable by any of its conjugates. This gives us first of all the possibility $\beta= \pm \alpha^{(i)}+h$. In the proof of the foregoing theorem we saw that $F_{K}(x, y)$ is of the form $a x^{3}+b x^{2} y+(b-3 a) x y^{2}-a y^{3}$. We shall compute the minimal polynomial of the number

$$
\beta_{0}=\left(t^{2}-s t+q+(t-s) \alpha+\alpha^{2}\right) / a=\left(t-\alpha^{\prime}\right)\left(t-\alpha^{\prime \prime}\right) / a
$$

appearing in (ii). We have $\operatorname{Irr}(\alpha, \mathbf{Q})=a^{-1} F_{K}(x-t,-e a)$ so that

$$
N\left(t-\alpha^{(i)}\right)=a^{-1} F_{K}(0,-e a)=e a^{3}
$$

for each $i$ and hence $N\left(\beta_{0}\right)=a^{3}$. A straightforward computation gives $\operatorname{Tr}\left(\beta_{0}\right)=$ $b-3 a$. From the condition $R\left(\beta_{0}\right)=T=9 a^{2}-3 a b+b^{2}$ we get the missing coefficient and the result is

$$
\operatorname{Irr}\left(\beta_{0}, \mathbf{Q}\right)=x^{3}-(b-3 a) x^{2}-a b x-a^{3}=a^{-1} F_{K}(x+a,-a) .
$$


Comparing the two minimal polynomials, we find

$$
\beta_{0}=e \alpha^{(i)}-a-e t \quad(i=1 \text { or } 2)
$$

because it is obvious that (5.13) cannot hold for $i=0$. Therefore, the numbers resulting from (ii) by replacing $\alpha$ by any of its conjugates are of the form $\pm \alpha^{(j)}+h$, where $h \in \mathbf{Z}$.

In the present case, (5.9) has the further solution $h_{11}=-1, h_{21}=1$. The solution with signs reversed is not acceptable because the leading coefficient $a_{1}(=a)$ of the form $F_{1}(x, y)$ must be positive. Since the substitution is unimodular, we have $h_{12}+h_{22}= \pm 1$. By interchanging conjugates of $\alpha$ if need be, we may take

$$
-x+h_{12} y-(e(t-\alpha) / a)\left(x+h_{22} y\right)=C\left(x+\left(\left(t_{1}-\beta\right) / a\right) y\right),
$$

where $C$ is a constant. It follows that $\beta= \pm \beta_{1}+h$ where $h \in \mathbf{Z}$ and

$$
\beta_{1}=-a^{2} /(a+e(t-\alpha)) \text {. }
$$

We have

$$
N(a+e(t-\alpha))=e N(e a+t-\alpha)=e a^{-1} F_{K}(e a,-e a)=-a^{3},
$$

so that

$$
\beta_{1}=\left(e a+t-\alpha^{\prime}\right)\left(e a+t-\alpha^{\prime \prime}\right) / a .
$$

Comparing this expression with (5.12) and using (5.13) we find

$$
\beta_{1}=\beta_{0}+e \alpha+a+e(2 t-s)=-e \alpha^{(j)}+e t \quad(j=1 \text { or } 2) .
$$

This proves (iii).

For practical purposes it is useful to know the approximate size of the coefficients of an NCP. The following theorem contains estimates to that effect. Numerical examples show that these estimates are rather accurate.

THEOREM 8. Let $f(x)=x^{3}-s x^{2}+q x-n$ be an NCP and let $D$ denote the discriminant of the corresponding field $K$. Then

$$
\begin{gathered}
s=0 \text { or } 1, \\
-D^{1 / 2} / 3+s / 3 \leqslant q<-(D / 4)^{1 / 3}+s / 3, \\
-(2 / 27) D^{3 / 4}-s D^{1 / 2} / 9<n<(2 / 27) D^{3 / 4} .
\end{gathered}
$$

Proof. The equality (5.14) holds trivially. Write $F_{K}(x, y)=a x^{3}+b x^{2} y+c x y^{2}+$ $d y^{3}$. From (4.4) and (3.8) we obtain the lower estimate (5.15). From (4.6) we have $27 D<4 T^{3}$, so that the upper estimate (5.15) also follows from (4.4). It is now easy to deduce (5.16) from (4.6) and (5.15).

6. The Algorithm. We shall now describe our algorithm which allows one to determine a complete set of NCP's whose zeros generate all totally real cubic fields of discriminant $D$ satisfying $X_{0}<D \leqslant X$, where $X_{0}$ and $X$ are given bounds. We start the algorithm by making a search for the relevant reduced forms. Following the classification in Theorem 1 , we first determine all integral vectors $(a, b, c, d)$ with $\operatorname{gcd}(a, b, c, d)=1$ which satisfy one of the following systems of conditions (where 
$\kappa=(35+13 \sqrt{13}) / 216$ as in the proof of Theorem 2$):$

$$
\begin{gathered}
1 \leqslant a<2 X^{1 / 4} / \sqrt{27}, \quad 1 \leqslant b \leqslant 2 X^{1 / 4} / \sqrt{3}, \\
\max \left\{-X^{1 / 2} / b,-(\kappa X / a)^{1 / 3},\left(b^{2}-X^{1 / 2}\right) /(3 a)\right\}<c \\
<\min \left\{X^{1 / 2} / b,(\kappa X / a)^{1 / 3},\left(b^{2}-\left(27 X_{0} a^{2} / 4\right)^{1 / 3}\right) /(3 a)\right\}, \\
\max \left\{(b c-T) /(9 a),\left(c^{2}-\left(3 X+T^{2}\right) /(4 T)\right) /(3 b)\right\}<d \\
<\min \left\{(b c+T) /(9 a),\left(c^{2}-T\right) /(3 b),\left(c^{2}-3 X_{0} /(4 T)\right) /(3 b)\right\} ; \\
1 \leqslant a<2 X^{1 / 4} / \sqrt{27}, \quad b=0, \\
\max \left\{-(\kappa X / a)^{1 / 3},-X^{1 / 2} /(3 a)\right\}<c<\min \left\{-3 a,-\left(X_{0} /(4 a)\right)^{1 / 3}\right\}, \\
1 \leqslant d<-c / 3 ; \\
\max \left\{-X^{1 / 2} / b,-(\kappa X / a)^{1 / 3},\left(b^{2}-X^{1 / 2}\right) /(3 a)\right\}<c \\
<\min \left\{b-3 a, X^{1 / 2} / b,(\kappa X / a)^{1 / 3},\left(b^{2}-\left(27 X_{0} a^{2} / 4\right)^{1 / 3}\right) /(3 a)\right\}, \\
d=(b c-T) /(9 a) ;
\end{gathered}
$$

(iii.1) $\quad 1 \leqslant a<2 X^{1 / 4} / \sqrt{27}, \quad 3 a / 2<b \leqslant 2 X^{1 / 4} / \sqrt{3}$,

$$
\max \{-3 a,-b\}<c<b-3 a, \quad d=\left(c^{2}-b^{2}+3 a c\right) /(3 b) ;
$$

$$
\begin{gathered}
1 \leqslant a<2 X^{1 / 4} / \sqrt{27}, \quad-2 X^{1 / 4} / \sqrt{3} \leqslant b<-3 a, \quad b<c<-3 a, \\
d=\left(c^{2}-b^{2}+3 a c\right) /(3 b) ;
\end{gathered}
$$

(iv) $\quad 1 \leqslant a<2 X^{1 / 4} / \sqrt{27}, \quad 3 a<b \leqslant 2 X^{1 / 4} / \sqrt{3}, \quad c=-3 a, \quad d=-b / 3$;

(v) $1 \leqslant a<2 X^{1 / 4} / \sqrt{27}, \quad 3 a / 2<b \leqslant 2 X^{1 / 4} / \sqrt{3}, \quad c=b-3 a, \quad d=-a$.

In deriving these conditions frequent use is made of (2.4), (2.5), (2.6) and (2.7). All bounds involving $X$ or $X_{0}$ are consequences of (3.1), (3.2), (3.3), (3.4) and (3.8). All other inequalities and equalities with two exceptions are critical in the sense that their validity is necessary and sufficient for the conditions of reduction to hold in each respective case. The exceptional ones are the bounds for $b$ in terms of $a$ in (iii.1) and (iii.2), which are consequences of the bounds for $c$. If a fractional expression appears in an equality it is of course stipulated that the numerator is divisible by the denominator. An overwhelming majority of reduced forms belongs to Case (i). It is therefore of minor practical importance to include all the possible alternative bounds for the various quantities in the above estimates in other cases, and we have not always done so.

The next step is to compute the value of the discriminant $D$ and to discard all forms which do not satisfy $X_{0}<D \leqslant X$ or the conditions (DH2), (DH3), (DHp) in Theorem 4. We then determine the associated polynomial $f(x)$ from (4.1) and (4.2) and compute its zeros. If any of these zeros is a rational integer, $f(x)$ is rejected.

In this way we have found the required complete set of NCP's. Let $f(x)=x^{3}-$ $s x^{2}+q x-n$ be any of these and let $K$ be the cubic field generated by a zero $\alpha$ of $f(x)$. As mentioned in the Introduction, in addition to leading to a naturally defined NCP, our approach has also practical advantages compared with the usual one. 
Firstly, there is no need to check whether fields of the same discriminant are the same or not. Secondly, we know that the discriminant $D$ of the form $F_{K}(x, y)$ computed above is also the discriminant of the field $K$. Furthermore, the leading coefficient $a$ of the form $F_{K}(x, y)$ satisfies (5.4) and $\mathcal{O}$ has an integral basis $\{1, \alpha, \omega\}$, where

$$
\begin{aligned}
& \omega=\left(l+m \alpha+\alpha^{2}\right) / a, \\
& l \equiv t^{2}-s t+q \bmod a, \quad m \equiv t-s \bmod a, \quad t=(s-e b) / 3, \\
& 0 \leqslant l<a, \quad 0 \leqslant m<a,
\end{aligned}
$$

in the notation (4.2).

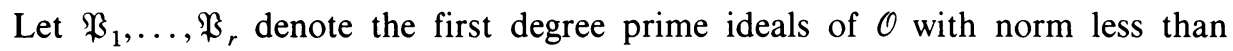
$D^{1 / 2} / 9.1$. These ideals are calculated and stored in a list. It follows from a result of Davenport [5] that every ideal class of $K$ contains a product of nonnegative powers of the ideals $\mathfrak{B}_{i}$. Here one has to take into account that there are only two cubic fields with discriminants $\leqslant 81$ which are exceptional and they both have class number $=1$. By virtue of the results of H. P. F. Swinnerton-Dyer [19] one could replace the constant 9.1 by a larger one, possibly at the cost of supplementary exceptional fields.

Applying the Voronoi algorithm, we determine the class number and the structure of the class group $G$ of the field $K$ inductively as follows. Suppose that for some $j$ $(1 \leqslant j \leqslant r)$ we already know the subgroup $H_{j-1}$ of $G$ generated by the classes represented by $\mathcal{O}$ and $\mathfrak{B}_{1}, \ldots, \mathfrak{B}_{j-1}$. We choose a representative for each element of $H_{j-1}$, consider it in a natural way as a lattice $\mathfrak{A}$ in $\mathbf{R}^{3}$, compute a $y$-chain of relative minima for $\mathfrak{A}[9$, Section 54], and calculate the lattices obtained by division of $\mathfrak{U}$ by elements of the $y$-chain within the bounds of one period. All these lattices are stored in a list called the comparison list. We then determine the smallest positive exponent $k$ such that there is a lattice obtained in a similar way from an $x$-chain for $\mathfrak{B}_{j}^{k}$ which is already contained in the comparison list [9, p. 258, Theorem 7]. If $k=1$, we have $H_{j}=H_{j-1}$. If $k>1$, then $H_{j}$ is generated by $H_{j-1}$ and the classes containing $\mathfrak{P}_{j}, \ldots, \mathfrak{B}_{j}^{k-1}$. The enlarged comparison list is computed in an obvious way. In order to avoid lattices with large generators we replace any ideal by an equivalent one encountered in the course of the computation if the latter has smaller norm than the previous one.

Let $\mathfrak{A}$ be any nonzero integral ideal of $K$. Suppose that the coordinate axes in $\mathbf{R}^{3}$ are chosen so that the vector corresponding to any number $\eta$ of $K$ is $\left(\eta, \eta^{\prime}, \eta^{\prime \prime}\right)$. (We shall use the same notation for such a vector and its $x$-coordinate). Let $\eta_{0}$ be a relative minimum in $\mathfrak{A}$ and let $\eta_{0}, \eta_{1}, \ldots$ be a chain of relative minima starting from $\eta_{0}$. For definiteness, suppose it is an $x$-chain. Write $\left|N\left(\eta_{n}\right)\right|=r_{n} N(\mathfrak{A})$ and take $\mathfrak{U}_{n}=\left(r_{n} / \eta_{n}\right) \mathfrak{A}$. Obviously, $r_{n}$ is a relative minimum in the lattice $\mathfrak{U}_{n}$, and if $\xi_{n+1}$ denotes the minimum point in this lattice adjacent to $r_{n}$ on the $x$-axis [9, p. 247], then

$$
\eta_{n+1}= \pm \eta_{n} \xi_{n+1} / r_{n} \quad(n=0,1, \ldots) .
$$

This is, of course, the principle on which the application of the Voronoi algorithm is based. At each step the computation is concerned with the determination of $\mathfrak{A}_{n}$ and the relative minimum $\xi_{n+1}$ adjacent to a given lattice point $r_{n}$ with equal conjugates.

Let $\{1, \alpha, \omega\}$ be the integral basis satisfying (6.1). It is of substantial practical importance to know the order of magnitude of the integer coordinates of $\xi_{n+1}$ with 
respect to this basis, i.e., the solution $x_{0}, x_{1}, x_{2}$ of

$$
\begin{gathered}
x_{0}+x_{1} \alpha+x_{2} \omega=\xi_{n+1}, \quad x_{0}+x_{1} \alpha^{\prime}+x_{2} \omega^{\prime}=\xi_{n+1}^{\prime}, \\
x_{0}+x_{1} \alpha^{\prime \prime}+x_{2} \omega^{\prime \prime}=\xi_{n+1}^{\prime \prime} .
\end{gathered}
$$

It follows directly from Minkowski's theorem that $r_{n} \leqslant D^{1 / 2}$. Since $\xi_{n+1}$ is adjacent to $r_{n}$ on the $x$-axis, we have $\left|\xi_{n+1}^{\prime}\right|<r_{n},\left|\xi_{n+1}^{\prime \prime}\right|<r_{n}$, and a repeated application of Minkowski's theorem gives $\left|\xi_{n+1}\right| \leqslant D^{1 / 2}$ because $N\left(\mathfrak{A}_{n}\right)=r_{n}^{2}$. Using Schwarz's inequality we get from (6.2)

$$
\begin{aligned}
D^{1 / 2}\left|x_{0}\right| & =\left|\xi_{n+1}\left(\alpha^{\prime} \omega^{\prime \prime}-\alpha^{\prime \prime} \omega^{\prime}\right)+\xi_{n+1}^{\prime}\left(\alpha^{\prime \prime} \omega-\alpha \omega^{\prime \prime}\right)+\xi_{n+1}^{\prime \prime}\left(\alpha \omega^{\prime}-\alpha^{\prime} \omega\right)\right| \\
& \leqslant \sqrt{3} D^{1 / 2}\left(\left(\alpha^{\prime} \omega^{\prime \prime}-\alpha^{\prime \prime} \omega^{\prime}\right)^{2}+\left(\alpha^{\prime \prime} \omega-\alpha \omega^{\prime \prime}\right)^{2}+\left(\alpha \omega^{\prime}-\alpha^{\prime} \omega\right)^{2}\right)^{1 / 2} \\
D^{1 / 2}\left|x_{1}\right| & =\left|\xi_{n+1}\left(\omega^{\prime}-\omega^{\prime \prime}\right)+\xi_{n+1}^{\prime}\left(\omega^{\prime \prime}-\omega\right)+\xi_{n+1}^{\prime \prime}\left(\omega-\omega^{\prime}\right)\right| \\
& \leqslant \sqrt{3} D^{1 / 2}\left(\left(\omega^{\prime}-\omega^{\prime \prime}\right)^{2}+\left(\omega^{\prime \prime}-\omega\right)^{2}+\left(\omega-\omega^{\prime}\right)^{2}\right)^{1 / 2} \\
D^{1 / 2}\left|x_{2}\right| & =\left|\xi_{n+1}\left(\alpha^{\prime \prime}-\alpha^{\prime}\right)+\xi_{n+1}^{\prime}\left(\alpha-\alpha^{\prime \prime}\right)+\xi_{n+1}^{\prime \prime}\left(\alpha^{\prime}-\alpha\right)\right| \\
& \leqslant \sqrt{3} D^{1 / 2}\left(\left(\alpha^{\prime \prime}-\alpha^{\prime}\right)^{2}+\left(\alpha-\alpha^{\prime \prime}\right)^{2}+\left(\alpha^{\prime}-\alpha\right)^{2}\right)^{1 / 2}
\end{aligned}
$$

Calculating the expressions for the symmetric functions in terms of $s, q, n, a, l, m$ and using (5.14), (5.15), (5.16), (6.1) we obtain, after a trivial but somewhat messy computation,

$$
\begin{aligned}
\left|x_{0}\right| \leqslant \sqrt{3} a^{-1}\left(l^{2}(2 s-6 q)+2 l\left(-s q+4 q^{2}-3 s n\right)\right. & \\
& \left.+(s-4 q)\left(q^{2}-2 s n\right)+2 s q n-9 n^{2}\right)^{1 / 2} \\
& <(2 / 3) D^{3 / 4}, \\
\left|x_{1}\right| & \leqslant \sqrt{3} a^{-1}\left((m+s)^{2}(2 s-6 q)-2(m+s)(s q-9 n)+2 q^{2}-6 s n\right)^{1 / 2} \\
& <\sqrt{6}\left(D^{1 / 2} / 3+D^{1 / 4}\right), \\
\left|x_{2}\right| & \leqslant(6 s-18 q)^{1 / 2} \leqslant \sqrt{6} D^{1 / 4} .
\end{aligned}
$$

In conclusion, we shall briefly sketch the determination of a fundamental pair of units by Voronoi's method. Take $\mathfrak{A}=\mathcal{O}, \eta_{0}=1$ and consider the $x$-chain $\eta_{0}, \eta_{1}, \ldots$ in $\mathfrak{U}$ as above. Let $\mathfrak{A}_{k}$ be the first repeating lattice, i.e., $\mathfrak{A}_{k}=\mathfrak{A}_{j}$ for some $0 \leqslant j<k$. Then $\varepsilon_{* 1}=\eta_{k} / \eta_{j}$ is the first fundamental unit. Next let $\zeta_{0}, \zeta_{1}, \ldots$ denote the $y$-chain in the lattice $\mathfrak{U}_{j-1}$ starting from $\zeta_{0}=\xi_{j}$ in the above notation. (If $j=0$ we take $\mathfrak{U}_{-1}=\mathcal{O}$ and $\zeta_{0}=1$.) Let $p$ be the smallest positive index for which an $h \in\{j, \ldots$, $k-1\}$ can be found such that there is an equality of lattices $\left(1 / \zeta_{p}\right) \mathfrak{A}_{j-1}=$ $\left(1 / \eta_{h}\right) \mathfrak{A}$. Then $\varepsilon_{* 2}=\left(\eta_{j} \zeta_{p}\right) /\left(\eta_{h} \zeta_{0}\right)$ is the second fundamental unit.

The two-dimensional unit lattice in the logarithmic space is generated by the vectors $\left(\log \left|\varepsilon_{*_{i}}\right|, \log \left|\varepsilon_{*_{i}}^{\prime}\right|, \log \left|\varepsilon_{*_{i}}^{\prime \prime}\right|\right) \quad(i=1,2)$. We compute a reduced fundamental parallelogram of this lattice by the usual process, i.e., we determine a fundamental pair of units $\varepsilon_{1}, \varepsilon_{2}$, called reduced units, so that the form

$$
\left(x \log \left|\varepsilon_{1}\right|+y \log \left|\varepsilon_{2}\right|\right)^{2}+\left(x \log \left|\varepsilon_{1}^{\prime}\right|+y \log \left|\varepsilon_{2}^{\prime}\right|\right)^{2}+\left(x \log \left|\varepsilon_{1}^{\prime \prime}\right|+y \log \left|\varepsilon_{2}^{\prime \prime}\right|\right)^{2}
$$

is a (semi) reduced positive definite binary quadratic form. There still is a free choice between $\pm \varepsilon_{i}^{ \pm 1}$. The choice is made so that $N\left(\varepsilon_{i}\right)=+1$ and two of the conjugates of 
$\varepsilon_{i}$ have absolute value $>1$. Presumably the order of magnitude of the coordinates of the pair $\varepsilon_{1}, \varepsilon_{2}$ with respect to the above basis $\{1, \alpha, \omega\}$ is near the least possible.

7. Tables and Statistics. The table containing the 26440 nonconjugate totally real cubic fields with discriminants less than 500000 has been deposited in the Mathematics of Computation's UMT-depository. It consists of 10 parts, the $k$ th part containing the fields with discriminants between $50000(k-1)$ and $50000 k$. For each field $K=\mathbf{Q}(\alpha)$, where $\alpha$ is an NPE the following data are listed:

- running number $r$

- discriminant $D$ of $K$

- coefficients $s, q, n$ of the polynomial $\operatorname{Irr}(\alpha, \mathbf{Q})=x^{3}-s x^{2}+q x-n$

- index $a$ satisfying (5.4)

- numbers $l, m$ satisfying (6.1) so that $\{1, \alpha, \omega\}$ with $\omega=\left(l+m \alpha+\alpha^{2}\right) / a$ is an integral basis for $\mathcal{O}$

- class number $h$ of $K$

- coefficients $a_{i j}(i=1,2 ; j=0,1,2)$ of the reduced units $\varepsilon_{i}=a_{i 0}+a_{i 1} \alpha+a_{i 2} \omega$

- TP indicates that every norm-positive unit is totally positive.

The data are listed in the format

$$
\begin{array}{llllllllll}
r & D & s & q & n & a & l & m & h & a_{i j}
\end{array}
$$

where the numbers $a_{i j}$ are arranged in the form of one of the following three matrices depending on the space occupied by them:

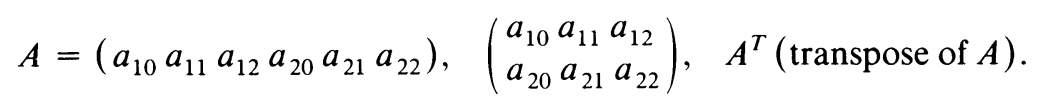

The program was constructed in FORTRAN 5A for the DEC-20 system at the Computer Centre of the University of Turku. The total CPU time required was about 55 hours. Most of the calculations were performed either in integral arithmetic or double-precision arithmetic with 18 significant digits. However, when computing the units one needs a precision of much higher order of magnitude. To begin with, we used an accuracy of 120 digits. The program contained various reliability checks. The sufficiency of the space reserved for the numbers was tested, and integers being expressed as a decimal were tested to have either 00000 or 99999 as first digits after the decimal point. Finally, the norms of the reduced units were checked for actually having the value 1 . If the field $K$ under consideration failed to pass the test, a warning index in the corresponding record was given a positive value. Afterwards, we gathered together all such faulty cases and repeated the computation using 250 digits. This was found to be sufficient with the exception of three large cases requiring about 300 digits. The necessary multi-precision routines were constructed by ourselves.

The table below gives statistics referring to the class numbers. The numbers at the top of each column are the bounds on the discriminant.

As remarked in the Introduction the corresponding table in [1] is erroneous and a revised version has been given by Llorente and Oneto [15]. However, even the latter is not fully in accordance with our results. In fact there are six differences; we have 
found the following numbers of occurrences:

$$
\begin{aligned}
& \text { for } 20001 \leqslant D \leqslant 30000 \text { and class number }= \begin{cases}1 & \text { no. }=414 \\
2 & \text { no. }=27\end{cases} \\
& \text { for } 30001 \leqslant D \leqslant 40000 \text { and class number }= \begin{cases}4 & \text { no. }=3 \\
5 & \text { no. }=3\end{cases} \\
& \text { for } 60001 \leqslant D \leqslant 70000 \text { and class number }= \begin{cases}2 & \text { no. }=33 \\
3 & \text { no. }=32\end{cases}
\end{aligned}
$$

\begin{tabular}{|c|c|c|c|c|c|}
\hline $\begin{array}{l}\text { Class } \\
\text { number }\end{array}$ & $\begin{array}{r}1 \\
50000 \\
\end{array}$ & $\begin{array}{r}50001 \\
100000 \\
\end{array}$ & $\begin{array}{l}100001 \\
150000 \\
\end{array}$ & $\begin{array}{l}150001 \\
200000 \\
\end{array}$ & $\begin{array}{l}200001 \\
250000 \\
\end{array}$ \\
\hline 1 & 2023 & 2169 & 2204 & 2204 & 2258 \\
\hline 2 & 109 & 181 & 193 & 199 & 230 \\
\hline 3 & 112 & 155 & 143 & 162 & 163 \\
\hline 4 & 11 & 10 & 17 & 20 & 29 \\
\hline 5 & 6 & 12 & 12 & 11 & 11 \\
\hline 6 & 1 & 6 & 11 & 12 & 17 \\
\hline 7 & 1 & 6 & - & 3 & 3 \\
\hline 8 & - & 1 & 4 & 3 & 2 \\
\hline 9 & - & 1 & 4 & 4 & 2 \\
\hline 10 & - & - & 2 & - & - \\
\hline 11 & - & - & - & 2 & - \\
\hline 12 & - & - & - & - & - \\
\hline 13 & - & - & 1 & - & - \\
\hline $\begin{array}{l}\text { Total no } \\
\text { of fields }\end{array}$ & 2263 & 2541 & 2591 & 2620 & 2715 \\
\hline
\end{tabular}

\begin{tabular}{|c|c|c|c|c|c|}
\hline $\begin{array}{l}\text { Class } \\
\text { number }\end{array}$ & $\begin{array}{l}250001 \\
300000\end{array}$ & $\begin{array}{r}300001 \\
350000 \\
\end{array}$ & $\begin{array}{l}350001 \\
400000 \\
\end{array}$ & $\begin{array}{l}400001 \\
450000 \\
\end{array}$ & $\begin{array}{l}450001 \\
500000 \\
\end{array}$ \\
\hline 1 & 2261 & 2244 & 2278 & 2309 & 2270 \\
\hline 2 & 216 & 232 & 238 & 229 & 232 \\
\hline 3 & 156 & 199 & 155 & 154 & 193 \\
\hline 4 & 26 & 24 & 26 & 29 & 32 \\
\hline 5 & 12 & 14 & 14 & 12 & 21 \\
\hline 6 & 10 & 16 & 9 & 17 & 11 \\
\hline 7 & 5 & 5 & 1 & 2 & 6 \\
\hline 8 & 1 & 3 & 2 & 3 & 2 \\
\hline 9 & 3 & 3 & 5 & 3 & 2 \\
\hline 10 & - & 3 & 1 & 3 & 1 \\
\hline 11 & - & 1 & - & - & - \\
\hline 12 & 1 & 1 & - & 5 & 3 \\
\hline 13 & - & - & 1 & - & 1 \\
\hline 14 & - & - & 1 & - & 1 \\
\hline 15 & - & 1 & - & - & - \\
\hline 16 & - & - & - & - & 1 \\
\hline$\left|\begin{array}{ll}\text { Total no } \\
\text { of fields }\end{array}\right|$ & 2691 & 2746 & 2731 & 2766 & 2776 \\
\hline
\end{tabular}


The following tables contain statistics about TP-cases and about nonconjugate fields with the same discriminant. Both these phenomena are of considerable theoretical interest.

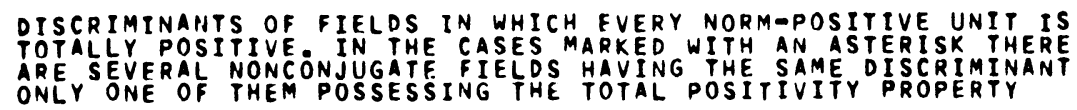

\begin{tabular}{|c|c|c|c|c|c|c|c|}
\hline $\begin{array}{r}15529 \\
59749 \\
97997 \\
126857 \\
155917 \\
194549 \\
225653 \\
241556 \\
282593 \\
303156 \\
325153 \\
344065 \\
357656 \\
392881 \\
424505 \\
437677 \\
458477\end{array}$ & $\begin{array}{r}25717 \\
66536 \\
99732 \\
142877 \\
166877 \\
194581 \\
227065 \\
242881 \\
283932 \\
305684 \\
328013 \\
345332 \\
362837 \\
393244 \\
426188 \\
438344 \\
464485\end{array}$ & $\begin{array}{r}25961 \\
74708 \\
104153 \\
147788 \\
167333 \\
197513 \\
228237 \\
244756 \\
285865 \\
307817 \\
330452 \\
345656 \\
364492 \\
413572 \\
426357 \\
439397 \\
474952\end{array}$ & $\begin{array}{r}28669 \\
82661 \\
109048 \\
148730 \\
171805 \\
206456 \\
230773 \\
249737 \\
289048 \\
313108 \\
333617 \\
348949 \\
375917 \\
404033 \\
428212 \\
446284 \\
479733\end{array}$ & $\begin{array}{r}29813 \\
86321 \\
109621 \\
149189 \\
174829 \\
206764 \\
230825 \\
260117 \\
292088 \\
317620 \\
336517 \\
349693 \\
377780 \\
405528 \\
429529 \\
448904 \\
482825\end{array}$ & $\begin{array}{r}37229 \\
88289 \\
114973 \\
150049 \\
176665 \\
215828 \\
236197 \\
270737 \\
294977 \\
318277 \\
336745 \\
350178 \\
380869 \\
415657 \\
429569 \\
450353 \\
485717\end{array}$ & $\begin{array}{r}53121 \\
94441 \\
119369 \\
152737 \\
184761 \\
219196 \\
237469 \\
276788 \\
295329 \\
321516 \\
338441 \\
353777 \\
386013 \\
420393 \\
430796 \\
451845 \\
488201\end{array}$ & $\begin{array}{r}57077 \\
95992 \\
124745 \\
154708 \\
189817 \\
225369 \\
24155 \\
277268 \\
297781 \\
324692 \\
341832 \\
353885 \\
387381\end{array}$ \\
\hline
\end{tabular}

DISCRIMINANTS WITH N ASSOCIATED NONCONJUGATE FIELOS FOR N $>1$

$N=2$

3969
46548
83700
149769
181300
213300
248724
278964
323028
363609
393492
441396
461041

8281
47089
90601
155412
182329
215700
255636
284148
327668
367956
395604
442260
465588

13689
55700
92340
157300
182868
215892
257556
296325
331425
370548
399924
452925
470988

17689
61009 110889

162324

185652

219961

259700

299700

334260

419796

456948
473300

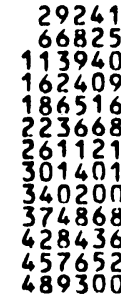

37300

67081
115668

164052

189972

231361

262964

302292

346921

43132

458325

489650

494209

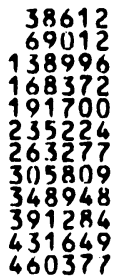

45684
77841

148372

208980

238140

275700

312481

3597012

435348

460404

$N=3$

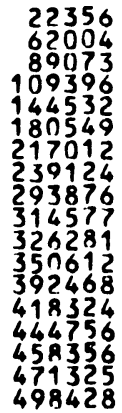

$\begin{array}{rr}28212 & 31425 \\ 62644 & 63028 \\ 95992 & 97844 \\ 119604 & 122300 \\ 146452 & 150164 \\ 189777 & 198045 \\ 223540 & 223604 \\ 239476 & 240692 \\ 295284 & 302612 \\ 314772 & 317300 \\ 326516 & 327537 \\ 334772 & 358425 \\ 394292 & 397300 \\ 419688 & 424148 \\ 444852 & 448092 \\ 459892 & 462537 \\ 476820 & 477981\end{array}$
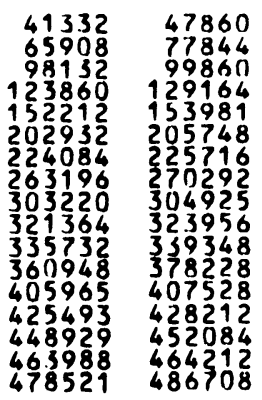

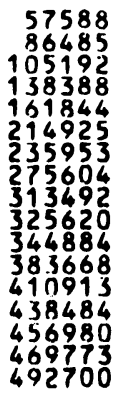

58077 86828 08729 144212 215796 236277 313620 325800 345716 384404 414708 439124 458260 470569

$\begin{array}{rr}32009 & 42817 \\ 142097 & 151141 \\ 206776 & 209765 \\ 252977 & 255973 \\ 320785 & 321053 \\ 363397 & 371965 \\ 424236 & 431761 \\ 476124 & 476152\end{array}$

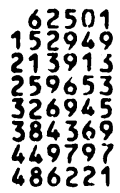

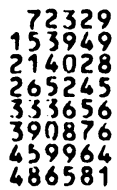

94636
172252
214712
275881
335229
400369
460817
494236
103809 173944 219461 341724 412277 468472

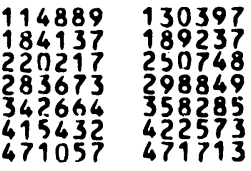


The notable increase in local density of these $N=3,4$ fields as $D$ increases, is discussed at length in [17] and is said to account for the increase in overall local density toward the theoretical limit $(12 \zeta(3))^{-1}$ that was mentioned above.

Our final table gives a list of discriminants $D$ and class numbers $h$ of fields with noncyclic class group. Each of these groups is generated by two elements. For the four cases with $h=12$ in the table there is more than one field with these $D$, but only one with a noncyclic group. On the other hand, both fields of $D=431649$ have noncyclic groups so they are listed twice. The frequency of occurrence of a noncyclic class group is 35 out of 26440 so that the phenomenon is a very uncommon one indeed. This is in conformity with the comprehensive results of Buell [4] on class groups of imaginary quadratic fields.

\begin{tabular}{crrrrr}
$D$ & $h$ & \multicolumn{1}{c}{$D$} & $h$ & \multicolumn{1}{c}{$D$} & $h$ \\
26569 & 4 & 35537 & 4 & 76729 & 4 \\
121801 & 4 & 128357 & 8 & 146853 & 9 \\
151717 & 4 & 157609 & 4 & 210649 & 4 \\
229577 & 4 & 240149 & 9 & 277429 & 4 \\
299209 & 4 & 312709 & 8 & 314369 & 4 \\
347485 & 4 & 368449 & 4 & 376712 & 9 \\
394609 & 4 & 395177 & 4 & 409533 & 4 \\
412277 & 12 & 424148 & 12 & 428657 & 8 \\
431649 & 9 & 431649 & 9 & 442489 & 4 \\
444412 & 8 & 455700 & 9 & 461041 & 12 \\
468892 & 8 & 474949 & 4 & 476249 & 4 \\
494209 & 12 & 496129 & 4 & &
\end{tabular}

Note added in March, 1984. After the completion of this work we have computed a supplementary table containing the values of the regulators up to $D<200000$. A table of unit signatures is in preparation.

Department of Mathematics

University of Turku

SF-20500 Turku 50

Finland

1. I. O. ANGell, "A table of totally real cubic fields," Math. Comp., v. 30, 1976, pp. 184-187. MR 53 \#5528

2. F. ARNDT, "Versuch einer Theorie der homogenen Funktionen des dritten Grades mit zwei Variabeln," Arch. Math. Phys., v. 17, 1851, pp. 1-53.

3. F. ARNDT, "Tabellarische Berechnung der reducirten binären kubischen Formen und Klassification derselben für alle successiven negativen Determinanten $(-D)$ von $D=3$ bis $D=2000$," Arch. Math. Phys., v. 31, 1858, pp. 335-448.

4. Duncan A. Buell, "Class groups of quadratic fields," Math. Comp., v. 30, 1976, pp. 610-623. MR 53 \# 8008

5. H. DavenPort, "On the product of three homogeneous linear forms. IV," Proc. Cambridge Philos. Soc., v. 39, 1943, pp. 1-21. MR 4, 212

6. H. DavenPort, "On the class-number of binary cubic forms (I)," J. London Math. Soc., v. 26. 1951, pp. 183-192; Corrigendum, v. 27, 1952, p. 512. MR 13, 323

7. H. Davenport \& H. Heilbronn, "On the density of discriminants of cubic fields," Bull. London Math. Soc., v. 1, 1969, pp. 345-348. MR $40 \# 7223$ 
8. H. Davenport \& H. Heilbronn, "On the density of discriminants of cubic fields. II," Proc. Roy. Soc. London Ser. A, v. 322, 1971, pp. 405-420. MR 58 \# 10816

9. B. N. Delone \& D. K. Faddeev, The Theory of Irrationalities of the Third Degree, Trudy Mat. Inst. Steklov., vol. 11, 1940; English transl., Transl. Math. Monographs, vol. 10, Amer. Math. Soc., Providence, R. I., Second printing 1978. MR 2, 349; 28 \# 3955

10. L. E. Dickson, History of the Theory of Numbers, vol. III, Carnegie Institution of Washington, Washington, 1923.

11. L. E. Dickson, Introduction to the Theory of Numbers, Dover, New York, 1957.

12. H. HASSE, "Arithmetische Bestimmung von Grundeinheit und Klassenzahl in zyklischen kubischen und biquadratischen Zahlkörpern," Abh. Deutsch. Akad. Wiss. Berlin, Math.-Nat. Kl., 1948, No. 2, 1950. MR 11, 503

13. Ch. Hermite, "Sur l'introduction des variables continues dans la théorie des nombres," J. Reine Angew. Math., v. 41, 1851, pp. 191-216; Oeuvres I, Gauthier-Villars, Paris, 1905, pp. 164-192.

14. Сн. Hermite, "Sur la réduction des formes cubiques a deux indéterminées," Comptes Rendus, v. 48, 1859, p. 351; Oeuvres II, Gauthier-Villars, Paris, 1908, pp. 93-99.

15. P. Llorente \& A. V. Oneto, "On the real cubic fields," Math. Comp., v. 39, 1982, pp. 689-692.

16. SIRPA MÄKI, The Determination of Units in Real Cyclic Sextic Fields, Lecture Notes in Math., vol. 797, Springer-Verlag, Berlin and New York, 1980. MR 82a: 12004

17. D. Shanks, Review of I. O. Angell, "A table of totally real cubic fields," Math. Comp., v. 30, 1976, pp. 670-673.

18. Rent Smadja, Calculs Effectifs sur les Ideaux des Corps de Nombres Algébriques, Université d'Aix-Marseille, U. E. R. Scientifique de Luminy, 1976.

19. H. P. F. Swinnerton-DYer, "On the product of three homogeneous linear forms," Acta Arith., v. 18, 1971, pp. 371-385. MR 45 \#1844. 